\title{
Quasi-Periodic Solutions in Nonlinear Asymmetric Oscillations
}

\author{
Xiaojing Yang and Kueiming Lo
}

Abstract. The existence of Aubry-Mather sets and infinitely many subharmonic solutions to the following $p$-Laplacian like nonlinear equation

$$
(p-1)^{-1}\left(\phi_{p}\left(x^{\prime}\right)\right)^{\prime}+\left[\alpha \phi_{p}\left(x^{+}\right)-\beta \phi_{p}\left(x^{-}\right)\right]+g(x)=h(t)
$$

is discussed, where $\phi_{p}(u)=|u|^{p-2} u, p>1, \alpha, \beta$ are positive constants satisfying $\alpha^{-\frac{1}{p}}+\beta^{-\frac{1}{p}}=\frac{2}{n}$ with $n \in \mathbb{N}, h$ is piece-wise two times differentiable and $2 \pi_{p}$-periodic, $g \in C^{1}(R)$ is bounded, $x^{ \pm}=\max \{ \pm x, 0\}, \pi_{p}=\frac{2 \pi}{p \sin (\pi / p)}$.

Keywords. Aubry-Mather sets, $p$-Laplacian, resonance, quasi-periodic solutions

Mathematics Subject Classification (2000). 34C25

\section{Introduction}

In this paper, we consider the existence of Aubry-Mather sets and quasi-periodic solutions to the following $p$-Laplacian like nonlinear differential equation

$$
\left.(p-1)^{-1}\left(\phi_{p}\left(x^{\prime}\right)\right)^{\prime}+\left[\alpha \phi_{p}\left(x^{+}\right)-\beta \phi_{p}\left(x^{-}\right)\right]+g(x)=h(t) \quad{ }^{\prime}=\frac{d}{d t}\right),
$$

where $\phi_{p}(u)=|u|^{p-2} u, p>1$ is a constant, $x^{ \pm}=\max \{ \pm x, 0\}, \alpha, \beta$ are positive constants satisfying

$$
\alpha^{-\frac{1}{p}}+\beta^{-\frac{1}{p}}=\frac{2}{n}
$$

$h$ is piece-wise two times differentiable and $2 \pi_{p}$-periodic, $g \in C^{1}(R)$ is bounded and $\pi_{p}=\frac{2 \pi}{p \sin (\pi / p)}$. If $p=2$, then $\pi_{2}=\pi$ and (1) reduces to second order differential equation

$$
x^{\prime \prime}+\alpha x^{+}-\beta x^{-}+g(x)=h(t) .
$$

Xiaojing Yang: Department of Mathematics, Tsinghua University, Beijing, 100084, China; yangxj@mail.tsinghua.edu.cn

Kueiming Lo: School of Software, Tsinghua University, Beijing 100084, China; gluo@tsinghua.edu.cn

This work is supported by the Funds 2004CB719400 and NSFC60474026. 
The existence of Aubry-Mather sets and quasi-periodic solutions of (3) was discussed recently in [3] if $g \in C^{2}$ and the limits

$$
\lim _{x \rightarrow+\infty} g(x)=g(+\infty), \quad \lim _{x \rightarrow-\infty} g(x)=g(-\infty)
$$

exist and $g$ satisfies some further approximate properties at infinity. Capietto and Liu [3], by applying a version of Aubry-Mather theory due to Pei [11], proved the existence of quasi-periodic solutions in generalized sense and multiplicity of subharmonic solutions to equation (3) under the so-called "resonance" situation, i.e., (2) holds for $p=2$ and some $n \in \mathbb{N}$.

Let $C$ be the solution of the initial value problem

$$
x^{\prime \prime}+\alpha x^{+}-\beta x^{-}=0, \quad x(0)=1, \quad x^{\prime}(0)=0 .
$$

Assume $\alpha \neq \beta$ and $\frac{1}{\sqrt{\alpha}}+\frac{1}{\sqrt{\beta}}=\frac{2 m}{n}, m, n \in \mathbb{N}$. Alonso and Ortega [2] proved that if the function

$$
\phi_{f}(\theta)=\int_{0}^{2 \pi} C\left(\frac{m \theta}{n}+t\right) h(t) d t
$$

has only simple zeros, then any solution $x$ of the linear equation

$$
x^{\prime \prime}+\alpha x^{+}-\beta x^{-}=h
$$

with large initial values, that is, if $\left|x\left(t_{0}\right)\right|+\left|x^{\prime}\left(t_{0}\right)\right| \gg 1$ for some $t_{0} \in \mathbb{R}$, goes to infinity in the future or in the past. Moreover, they showed the existence of $h$ such that unbounded solutions and $2 \pi$-periodic solutions of (4) can coexist.

Fabry and Fonda [4] and Fabry and Mawhin [5] obtained, by degree methods, sufficient conditions for the existence of $2 \pi$-periodic solutions and of unbounded solutions as well as subharmonic solutions for (5) below, respectively. More precisely, in [5] the following equation is considered:

$$
x^{\prime \prime}+\alpha x^{+}-\beta x^{-}=g(x)+f(x)+h(t),
$$

and it is proved that if the function

$$
\Phi_{h}(\theta)=\frac{n}{\pi}\left[\frac{g(+\infty)}{\alpha}-\frac{g(-\infty)}{\beta}\right]+\frac{1}{2 \pi \sqrt{\alpha}} \int_{0}^{2 \pi} C(t+\theta) h(t) d t
$$

has zeros and all of them are simple, then all solutions of (5) with large initial values are unbounded if the following resonance condition is satisfied:

$$
\frac{1}{\sqrt{\alpha}}+\frac{1}{\sqrt{\beta}}=\frac{2}{n}, \quad n \in \mathbb{N}
$$


and $f$ has a sublinear primitive, that is, $\lim _{|x| \rightarrow \infty} \frac{1}{x} \int_{0}^{x} f(s) d s=0$. Later, the author of this paper [12] discussed the more general equation (1) and considered the following function:

$$
\phi(\theta)=D_{p}-\frac{p}{\alpha^{\frac{1}{p}}} \int_{0}^{2 \pi_{p}} h(m t) C_{p}(m t+\theta) d t,
$$

where

$$
D_{p}=\frac{2 n}{m} B\left(\frac{2}{p}, 1-\frac{1}{p}\right)\left[\frac{g(+\infty)}{\alpha^{\frac{2}{p}}}-\frac{g(-\infty)}{\beta^{\frac{2}{p}}}\right],
$$

$B(r, s)$ is the $\beta$-function $B(r, s)=\int_{0}^{1}(1-t)^{r-1} t^{s-1} d t$ for $r>0, s>0$, and $C_{p}(t)$ is the $\frac{2 \pi_{p} m}{n}$-periodic solution of the following initial value problem:

$$
(p-1)^{-1}\left(\phi_{p}\left(u^{\prime}\right)\right)^{\prime}+\left[\alpha \phi_{p}\left(u^{+}\right)-\beta \phi_{p}\left(u^{-}\right)\right]=0, \quad u(0)=1, u^{\prime}(0)=0,
$$

if $\alpha$ and $\beta$ satisfy $\alpha^{-\frac{1}{p}}+\beta^{-\frac{1}{p}}=\frac{2 m}{n}, m, n \in \mathbb{N}$.

It was shown in [12] that if the function $\phi(\theta)$ has no zero for all $\theta \in \mathbb{R}$, then all solutions of (1) are bounded. For more recent results on boundedness and existence $2 \pi$-periodic solutions of (1) and (3), we refer [1], [6]-[9], [11]-[15] and the references therein.

In the rest of this paper, we denote by $S$ the unique solution of the initial value problem

$$
(p-1)^{-1}\left(\phi_{p}\left(x^{\prime}\right)\right)^{\prime}+\left[\alpha \phi_{p}\left(x^{+}\right)-\beta \phi_{p}\left(x^{-}\right)\right]=0, \quad x(0)=0, \quad x^{\prime}(0)=1 .
$$

\section{Definition 1.}

(A) A solution of $\left(x_{\omega}(t), x_{\omega}^{\prime}(t)\right)$ of (1) is called of Mather type with rotation number $\omega$ if $\omega=\frac{k}{m}$ is rational, the solutions $\left(x_{\omega}(t+2 i \pi), x_{\omega}^{\prime}(t+2 i \pi)\right)$, $1 \leq i \leq m-1$, are mutually unlinked periodic solutions of periodic $2 m \pi$ and, in this case,

$$
\lim _{\omega \rightarrow n} \min _{t \in \mathbb{R}}\left(\left|x_{\omega}(t)\right|+\left|x_{\omega}^{\prime}(t)\right|\right)=+\infty
$$

(B) If $\omega$ is irrational, the solution $\left(x_{\omega}(t), x_{\omega}^{\prime}(t)\right)$ is either a usual quasi-periodic solution or a generalized one, that is, the closed set

$$
\overline{\left\{\left(x_{\omega}(2 i \pi), x_{\omega}^{\prime}(2 i \pi)\right), i \in \mathbb{Z}\right\}}
$$

is a Denjoy's minimal set.

The main results of this paper are formulated in the following theorems: 
Theorem 1. Assume $h \in L^{\infty}\left[0,2 \pi_{p}\right]$ is $2 \pi_{p}$-periodic and $g \in C^{1}(\mathbb{R})$ is bounded and satisfies the following conditions: the limits $\lim _{x \rightarrow+\infty} g(x)=g(+\infty)$ and $\lim _{x \rightarrow-\infty} g(x)=g(-\infty)$ exist and $g$ satisfies

$$
g(x)=g( \pm \infty)+c^{ \pm}|x|^{-(p-1) \sigma} \operatorname{sgn} x+O\left(|x|^{-(p-1) \sigma-1}\right)
$$

for $|x| \gg 1$, where $\sigma \in\left(0, \frac{1}{p-1}\right)$ is a constant and $c^{ \pm}$are constants satisfying

$$
D_{0}=: \frac{c^{+}}{\alpha^{\frac{2-(p-1) \sigma}{p}}}+\frac{c^{-}}{\beta^{\frac{2-(p-1) \sigma}{p}}} \neq 0 .
$$

Define a $2 \pi_{p}$-periodic function $\lambda_{1}$ as

$$
\lambda_{1}(t)=\int_{0}^{2 \pi_{p}} S(\theta) h(t+\theta) d \theta-\frac{2}{p}\left(\frac{g(+\infty)}{\alpha^{\frac{2}{p}}}-\frac{g(-\infty)}{\beta^{2 / p}}\right) B\left(\frac{2}{p}, \frac{1}{q}\right),
$$

where $q=\frac{p}{p-1}$ is the conjugate exponent of $p$. Let one of the following conditions be satisfied:

(I) $\lambda_{1}(t) \neq 0$ for all $t \in \mathbb{R}$;

(II) either (a) $\lambda_{1}(t) \geq 0$ and $D_{0}<0$ or (b) $\lambda_{1}(t) \leq 0$ and $D_{0}>0$.

Then there exists an $\varepsilon_{0}>0$ such that for any $\omega \in\left(n, n+\varepsilon_{0}\right)$, equation (1) has a solution $\left(x_{\omega}(t), x_{\omega}^{\prime}(t)\right)$ of Mather type with rotation number $\omega$.

Theorem 2. Assume $g(x) \equiv 0, h$ is piece-wise two times differentiable and $2 \pi_{p}$-periodic. Assume

$$
\lambda_{1}(t)=\int_{0}^{2 \pi_{p}} S(\theta) h(t+\theta) d \theta \equiv 0 .
$$

For $p \neq 2$, define a $2 \pi_{p}$-periodic function $\lambda_{2}(t)$ as

$\lambda_{2}(t)=(p-2)\left[\int_{0}^{2 \pi_{p}} S(\theta) h(t+\theta) \int_{0}^{\theta} S(\tau) h^{\prime}(t+\tau) d \tau d \theta-\int_{0}^{2 \pi_{p}} S^{2}(\theta) h^{2}(t+\theta) d \theta\right]$.

For $p=2$, define a $2 \pi$-periodic function $\lambda_{3}(t)$ as

$$
\begin{aligned}
\lambda_{3}(t)= & -\frac{1}{2}\left[\int_{0}^{2 \pi} S^{3}(\theta) h^{3}(t+\theta) d \theta+\int_{0}^{2 \pi} S(\theta) h^{\prime \prime}(t+\theta)\left(\int_{0}^{\theta} S(\tau) h(t+\tau) d \tau\right)^{2} d \theta\right] \\
& -\int_{0}^{2 \pi} S^{2}(\theta) h(t+\theta) h^{\prime}(t+\theta) \int_{0}^{\theta} S(\tau) h(t+\tau) d \tau d \theta .
\end{aligned}
$$

Then the conclusions of Theorem 1 are true, if one of the following conditions holds:

(I) $p \neq 2, \lambda_{2}(t) \neq 0$ for all $t \in \mathbb{R}$;

(II) $p=2, \lambda_{3}(t) \neq 0$ for all $t \in \mathbb{R}$. 


\section{Generalized polar coordinates transformation}

If we introduce a new variable $y=\phi_{p}\left(x^{\prime}\right)$, then $(1)$ is equivalent to the planar system

$$
x^{\prime}=\phi_{q}(y), \quad y^{\prime}=(p-1)\left[-\alpha \phi_{p}\left(x^{+}\right)+\beta \phi_{p}\left(x^{-}\right)+h(t)-g(x)\right],
$$

where $q=\frac{p}{p-1}$ is the conjugate exponent of $p$. Let $u=\sin _{p} t$ be the solution of the initial value problem

$$
\left(\phi_{p}\left(u^{\prime}\right)\right)^{\prime}+(p-1) \phi_{p}(u)=0, \quad u(0)=0, u^{\prime}(0)=1
$$

which for $t \in\left[0, \frac{\pi_{p}}{2}\right]$ can be expressed implicitly by

$$
t=\int_{0}^{\sin _{p} t} \frac{d s}{\left(1-s^{p}\right)^{\frac{1}{p}}} .
$$

Then it follows from [10] that $u=\sin _{p} t$ can be extended to $\mathbb{R}$ as a $2 \pi_{p}$-periodic odd $C^{2}$-function which satisfies $\sin _{p} t:\left[0, \frac{\pi_{p}}{2}\right] \rightarrow[0,1]$ and $\sin _{p}\left(\pi_{p}-t\right)=\sin _{p} t$ for $t \in\left[\frac{\pi_{p}}{2}, \pi_{p}\right], \sin _{p}\left(2 \pi_{p}-t\right)=-\sin _{p} t$ for $t \in\left[\pi_{p}, 2 \pi_{p}\right]$.

Let the function $S$ be the unique solution of problem (6), then it is not difficult to verify that $S \in C^{2}(R)$ is $\frac{2 \pi_{p}}{n}$-periodic and can be expressed as

$$
S(t)=\left\{\begin{array}{cl}
\alpha^{-\frac{1}{p}} \sin _{p} \alpha^{\frac{1}{p}} t, & t \in\left[0, \alpha^{-\frac{1}{p}} \pi_{p}\right) \\
-\beta^{-\frac{1}{p}} \sin _{p} \beta^{\frac{1}{p}}\left(t-\alpha^{-\frac{1}{p}} \pi_{p}\right), & t \in\left[\alpha^{-\frac{1}{p}} \pi_{p}, \frac{2 \pi_{p}}{n}\right],
\end{array}\right.
$$

from which it is easy to verify that the following equality holds:

$$
\left|S^{\prime}(t)\right|^{p}+\alpha\left(S^{+}(t)\right)^{p}+\beta\left(S^{-}(t)\right)^{p} \equiv 1, \quad t \in R .
$$

For $\rho>0, \theta\left(\bmod 2 \pi_{p}\right)$, we define the generalized polar coordinates transformation $(\rho, \theta) \rightarrow(x, y)$ as

$$
x=\rho^{\frac{1}{p}} S\left(\frac{\theta}{n}\right), \quad y=\rho^{\frac{1}{q}} \phi_{p}\left(S^{\prime}\left(\frac{\theta}{n}\right)\right) .
$$

Under this transformation and by using (9), (7) is changed into the planar system

$$
\rho^{\prime}=p \rho^{\frac{1}{p}} S^{\prime}\left(\frac{\theta}{n}\right)(h(t)-g(x)), \quad \theta^{\prime}=n-n \rho^{-\frac{1}{q}} S\left(\frac{\theta}{n}\right)(h(t)-g(x)) .
$$

If we define $r=\rho^{\frac{1}{q}}$, then (10) can be further simplified as

$$
r^{\prime}=(p-1) S^{\prime}\left(\frac{\theta}{n}\right)(h(t)-g(x)), \quad \theta^{\prime}=n\left[1-r^{-1} S\left(\frac{\theta}{n}\right)(h(t)-g(x))\right],
$$

where $x=r^{\frac{1}{p-1}} S\left(\frac{\theta}{n}\right)$. 
Let $\left(r\left(t ; r_{0}, \theta_{0}\right), \theta\left(t ; r_{0}, \theta_{0}\right)\right)$ be the solution of $(11)$ with initial value $\left(r_{0}, \theta_{0}\right)$. Then for large initial value, i.e., $r_{0} \gg 1$, by the boundedness of $h, g, S, S^{\prime}$ and for $t$ in any bounded interval $I \subset\left[0,2 n \pi_{p}\right]$, we get $r(t)=r_{0}+O(1)$ which yields $r^{-1}(t)=r_{0}^{-1}+O\left(r_{0}^{-2}\right)$. Going back to (11), we get for $t \in I, \theta^{\prime}(t) \geq \frac{1}{2}>0$. As in [3], we can write (11) in the following equivalent form:

$$
\begin{aligned}
& \frac{d t}{d \theta}=\frac{1}{n\left(1-r^{-1} S\left(\frac{\theta}{n}\right)(h(t)-g(x))\right.} \\
& \frac{d r}{d \theta}=\frac{(p-1) S^{\prime}\left(\frac{\theta}{n}\right)(h(t)-g(x))}{n\left(1-r^{-1} S\left(\frac{\theta}{n}\right)(h(t)-g(x))\right.} .
\end{aligned}
$$

Now let $\left(r\left(\theta ; r_{0}, t_{0}\right), t\left(\theta ; r_{0}, t_{0}\right)\right)$ be the solution of $(12)$ with initial value $\left(r_{0}, t_{0}\right)$ where $t_{0} \in I$ and $\theta \in\left[0,2 n \pi_{p}\right]$. Then for $r_{0} \gg 1$, we obtain $r(\theta) \geq r_{0} / 2 \gg 1$ and (12) can be written as

$$
\begin{aligned}
& \frac{d r}{d \theta}=\frac{p-1}{n}\left[S^{\prime}\left(\frac{\theta}{n}\right)(h(t)-g(x))+r^{-1}(\theta) S^{\prime}\left(\frac{\theta}{n}\right) S\left(\frac{\theta}{n}\right)(h(t)-g(x))^{2}+\cdots\right] \\
& \frac{d t}{d \theta}=\frac{1}{n}\left[1+r^{-1}(\theta) S\left(\frac{\theta}{n}\right)(h(t)-g(x))+r^{-2}(\theta) S^{2}\left(\frac{\theta}{n}\right)(h(t)-g(x))^{2}+\cdots\right]
\end{aligned}
$$

where $x=x(\theta)=r_{0}^{\frac{1}{p-1}} S\left(\frac{\theta}{n}\right)+O(1)$.

\section{Lemmas}

For the proof of theorems, we need the following lemmas:

Lemma 1. Assume the conditions of Theorem 1 hold, then we have

$$
\begin{aligned}
& r_{1}=r_{0}+\mu_{0}\left(t_{0}\right)+O\left(r_{0}^{-1}\right) \\
& t_{1}=t_{0}+2 \pi_{p}+\lambda_{1}\left(t_{0}\right) r_{0}^{-1}+\lambda_{1+\sigma} r_{0}^{-(1+\sigma)}+O\left(r_{0}^{-2}\right),
\end{aligned}
$$

where $r_{1}=r\left(2 n \pi_{p} ; r_{0}, t_{0}\right), t_{1}=t\left(2 n \pi_{p} ; r_{0}, t_{0}\right)$ and

$$
\begin{aligned}
\mu_{0}(t) & =(p-1) \int_{0}^{2 \pi_{p}} S^{\prime}(\theta) f(t+\theta) d \theta \\
\lambda_{1}(t) & =\int_{0}^{2 \pi_{p}} S(\theta) f(t+\theta) d \theta-\frac{2}{p}\left(\frac{g(+\infty)}{\alpha^{\frac{2}{p}}}-\frac{g(-\infty)}{\beta^{\frac{2}{p}}}\right) B\left(\frac{2}{p}, \frac{1}{q}\right) \\
\lambda_{1+\sigma} & =-\frac{2}{p}\left(\frac{c^{+}}{\alpha^{\frac{\tau+1}{p}}}+\frac{c^{-}}{\beta^{\frac{\tau+1}{p}}}\right) B\left(\frac{\tau+1}{p}, \frac{1}{q}\right) \\
& =-D_{0} \frac{2}{p} B\left(\frac{\tau+1}{p}, \frac{1}{q}\right),
\end{aligned}
$$

where $\tau=1-(p-1) \sigma \in(0,1)$. Moreover, we have $\mu_{0}(t)=-(p-1) \lambda_{1}^{\prime}(t)$. 
Proof. It follows from (13) and for $t_{0} \in \mathbb{R}$ and $\theta \in\left[0,2 n \pi_{p}\right]$, we have

$$
r(\theta)=r_{0}+O(1), \quad t(\theta)=t_{0}+\frac{\theta}{n}+O\left(r_{0}^{-1}\right) .
$$

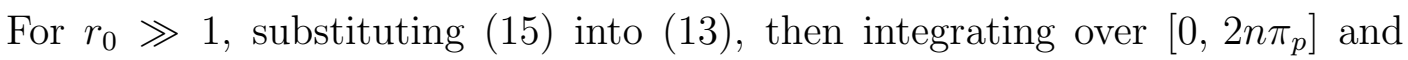
letting $r_{1}=r\left(2 n \pi_{p}\right), t_{1}=t\left(2 n \pi_{p}\right)$, we obtain

$$
\begin{aligned}
& r_{1}=r_{0}+\mu_{0}\left(t_{0}\right)+O\left(r_{0}^{-1}\right) \\
& t_{1}=t_{0}+2 \pi_{p}+\lambda_{1}\left(t_{0}\right) r_{0}^{-1}+\lambda_{1+\sigma} r_{0}^{-(1+\sigma)}+O\left(r_{0}^{-2}\right),
\end{aligned}
$$

where

$$
\begin{aligned}
\mu_{0}(t)= & \frac{p-1}{n} \int_{0}^{2 n \pi_{p}} S^{\prime}\left(\frac{\theta}{n}\right) h\left(t+\frac{\theta}{n}\right) d \theta \\
& -\frac{p-1}{n} g(+\infty) \int_{I} S^{\prime}\left(\frac{\theta}{n}\right) d \theta-\frac{p-1}{n} g(-\infty) \int_{J} S^{\prime}\left(\frac{\theta}{n}\right) d \theta \\
= & (p-1) \int_{0}^{2 \pi_{p}} S^{\prime}(\theta) h(t+\theta) d \theta,
\end{aligned}
$$

and

$$
\begin{aligned}
\lambda_{1}(t) & =\frac{1}{n}\left[\int_{0}^{2 n \pi_{p}} S\left(\frac{\theta}{n}\right) h\left(t+\frac{\theta}{n}\right) d \theta-g(+\infty) \int_{I} S\left(\frac{\theta}{n}\right) d \theta\right]-\frac{1}{n} g(-\infty) \int_{J} S\left(\frac{\theta}{n}\right) d \theta \\
& =\int_{0}^{2 \pi_{p}} S(\theta) h(t+\theta) d \theta-g(+\infty) \int_{0}^{\frac{\pi_{p}}{\alpha^{1 / p}}} S(\theta) d \theta+g(-\infty) \int_{\frac{\pi_{p}}{\alpha^{1 / p}}}^{\frac{2 \pi_{p}}{n}}|S(\theta)| d \theta
\end{aligned}
$$

where $I=\left\{\theta \in\left[0,2 n \pi_{p}\right]: S\left(\frac{\theta}{n}\right)>0\right\}$ and $J=\left\{\theta \in\left[0,2 n \pi_{p}\right]: S\left(\frac{\theta}{n}\right)<0\right\}$.

By using the similar method used in [12], we can show that

$$
\begin{aligned}
\int_{0}^{\frac{\pi_{p}}{\alpha^{1 / p}}} S(\theta) d \theta & =\frac{1}{\alpha^{\frac{2}{p}}} \frac{2}{p} B\left(\frac{2}{p}, \frac{1}{q}\right) \\
\int_{\alpha^{1 / p}}^{\frac{2 \pi_{p}}{n}} S(\theta) d \theta & =\frac{1}{\beta^{\frac{2}{p}}} \frac{2}{p} B\left(\frac{2}{p}, \frac{1}{q}\right) .
\end{aligned}
$$

From above equalities, we obtain the expressions of $\mu_{1}(t)$ and $\lambda_{1}(t)$.

Next, we calculate the value $\lambda_{1+\sigma}$. From (16) and the expression of $S$ in (8), we obtain

$$
\begin{aligned}
\lambda_{1+\sigma} & =-c^{+} \int_{0}^{\frac{\pi_{p}}{\alpha^{1 / p}}}(S(\theta))^{\tau} d \theta-c^{-} \int_{\frac{\pi_{p}}{\alpha^{1 / p}}}^{\frac{2 \pi_{p}}{n}}|S(\tau)|^{\tau} d \theta \\
& =-\left(\frac{c^{+}}{\alpha^{\frac{\tau+1}{p}}}+\frac{c^{-}}{\beta^{\frac{\tau+1}{p}}}\right) \int_{0}^{\pi_{p}}\left(\sin _{p} \theta\right)^{\tau} d \theta
\end{aligned}
$$


and

$$
\int_{0}^{\pi_{p}}\left(\sin _{p} \theta\right)^{\tau} d \theta=2 \int_{0}^{\frac{\pi_{p}}{2}}\left(\sin _{p} \theta\right)^{\tau} d \theta=\frac{2}{p} B\left(\frac{\tau+1}{p}, \frac{1}{q}\right),
$$

which yields the expression of $\lambda_{1+\sigma}$. Now the integration by parts yields $\mu_{0}(t)=$ $-(p-1) \lambda_{1}(t)$.

Lemma 2. Assume the conditions of Theorem 2 hold, then we have

$$
\begin{aligned}
& r_{1}=r_{0}+\mu_{1}\left(t_{0}\right) r_{0}^{-1}+O\left(r_{0}^{-2}\right) \\
& t_{1}=t_{0}+2 \pi_{p}+\lambda_{2}\left(t_{0}\right) r_{0}^{-2}+O\left(r_{0}^{-3}\right),
\end{aligned}
$$

where

$$
\begin{aligned}
\mu_{1}(t)= & -(p-1) \int_{0}^{2 \pi_{p}} S(\theta) h^{\prime \prime}(t+\theta) \int_{0}^{\theta} S(\tau) h(t+\tau) d \tau d \theta \\
& -2(p-1) \int_{0}^{2 \pi_{p}} S^{2}(\theta) h(t+\theta) h(t+\theta) d \theta \\
\lambda_{2}(t)= & (p-2) \int_{0}^{2 \pi_{p}} S(\theta) h^{\prime}(t+\theta) \int_{0}^{\theta} S(\tau) h(t+\tau) d \tau d \theta \\
& -(p-2) \int_{0}^{2 \pi_{p}} S^{2}(\theta) h^{2}(t+\theta) d \theta
\end{aligned}
$$

Moreover, we have $(p-2) \mu_{1}(t)=(p-1) \lambda_{2}^{\prime}(t)$.

Proof. Substituting (15) into (13) and integrating over $[0, \theta] \subset\left[0,2 \pi_{p}\right]$ we, obtain

$$
\begin{aligned}
r(\theta) & =r_{0}+\mu_{0}\left(t_{0}, \theta\right)+O\left(r_{0}^{-1}\right) \\
t(\theta) & =t_{0}+\frac{\theta}{n}+\lambda_{1}\left(t_{0}, \theta\right) r_{0}^{-1}+O\left(r_{0}^{-2}\right) \\
r^{-1}(\theta) & =r_{0}^{-1}-\mu_{0}\left(t_{0}, \theta\right) r_{0}^{-2}+O\left(r_{0}^{-3}\right),
\end{aligned}
$$

where

$$
\begin{aligned}
& \mu_{0}(t, \theta)=\frac{p-1}{n} \int_{0}^{\theta} S^{\prime}\left(\frac{\tau}{n}\right) h\left(t+\frac{\tau}{n}\right) d \tau \\
& \lambda_{1}(t, \theta)=\frac{1}{n} \int_{0}^{\theta} S\left(\frac{\tau}{n}\right) h\left(t+\frac{\tau}{n}\right) d \tau .
\end{aligned}
$$

Substituting (18)-(19) into (13) and integrating over [0, $\left.2 n \pi_{p}\right]$, we get

$$
\begin{aligned}
& r_{1}=r_{0}+\mu_{0}\left(t_{0}\right)+\mu_{1}\left(t_{0}\right) r_{0}^{-1}+O\left(r_{0}^{-2}\right) \\
& t_{1}=t_{0}+2 \pi_{p}+\lambda_{1}\left(t_{0}\right) r_{0}^{-1}+\lambda_{2}\left(t_{0}\right) r_{0}^{-2}+O\left(r_{0}^{-3}\right),
\end{aligned}
$$


where $\lambda_{1}(t)=\lambda_{1}\left(t, 2 n \pi_{p}\right), \mu_{0}(t)=\mu_{0}\left(t, 2 n \pi_{p}\right)$,

$$
\begin{aligned}
\mu_{1}(t)= & \frac{p-1}{n} \int_{0}^{2 n \pi_{p}} S^{\prime}\left(\frac{\theta}{n}\right) h^{\prime}\left(t+\frac{\theta}{n}\right) \lambda_{1}(t, \theta) d \theta \\
& +\frac{p-1}{n} \int_{0}^{2 n \pi_{p}} S\left(\frac{\theta}{n}\right) S^{\prime}\left(\frac{\theta}{n}\right) h^{2}\left(t+\frac{\theta}{n}\right) d \theta \\
= & -(p-1) \int_{0}^{2 \pi_{p}} S(\theta) h^{\prime \prime}(t+\theta) \int_{0}^{\theta} S(\tau) h(t+\tau) d \tau d \theta \\
& -2(p-1) \int_{0}^{2 \pi_{p}} S^{2}(\theta) h(t+\theta) h^{\prime}(t+\theta) d \theta-(p-1) \lambda_{1}(t) \lambda_{1}^{\prime}(t)
\end{aligned}
$$

and

$$
\begin{aligned}
\lambda_{2}(t)= & \frac{1}{n} \int_{0}^{2 n \pi_{p}} S^{2}\left(\frac{\theta}{n}\right) h^{2}\left(t+\frac{\theta}{n}\right) d \theta-\frac{1}{n} \int_{0}^{2 n \pi_{p}} S\left(\frac{\theta}{n}\right) h\left(t+\frac{\theta}{n}\right) \mu_{0}(t, \theta) d \theta \\
& +\frac{1}{n} \int_{0}^{2 n \pi_{p}} S\left(\frac{\theta}{n}\right) h^{\prime}\left(t+\frac{\theta}{n}\right) \lambda_{1}(t, \theta) d \theta \\
= & (p-2) \int_{0}^{2 \pi_{p}} S(\theta) h(t+\theta) \int_{0}^{\theta} S(\tau) h^{\prime}(t+\tau) d \tau d \theta \\
& -(p-2) \int_{0}^{2 \pi_{p}} S^{2}(\theta) h^{2}(t+\theta) d \theta+\lambda_{1}(t) \lambda_{1}^{\prime}(t)
\end{aligned}
$$

From above equalities we obtain after some elementary calculation

$$
(p-2) \mu_{1}(t)=(p-1)\left[\lambda_{2}^{\prime}(t)-\frac{p}{2}\left(\lambda_{1}^{\prime}(t)\right)^{2}-(p-1) \lambda_{1}(t) \lambda_{1}^{\prime \prime}(t)\right]
$$

which implies that, for $\lambda_{1}(t) \equiv 0$, we have $(p-2) \mu_{1}(t)=(p-1) \lambda_{2}^{\prime}(t)$.

Lemma 3. Assume that the conditions of Theorem 2 hold, and $p=2$. Then

$$
\begin{aligned}
& r_{1}=r_{0}+\mu_{1}\left(t_{0}\right) r_{0}^{-1}+O\left(r_{0}^{-2}\right) \\
& t_{1}=t_{0}+2 \pi+\lambda_{3}\left(t_{0}\right) r_{0}^{-3}+O\left(r_{0}^{-4}\right),
\end{aligned}
$$

where

$\mu_{1}(t)=-\int_{0}^{2 \pi} S(\theta) h^{\prime \prime}(t+\theta) \int_{0}^{\theta} S(\tau) h(t+\tau) d \tau d \theta-2 \int_{0}^{2 \pi} S^{2}(\theta) h(t+\theta) h^{\prime}(t+\theta) d \theta$ and $\lambda_{3}(t)$ is given as in Theorem 2 .

Proof. Substituting (15) into (13) and integrating over $[0, \theta] \subset[0,2 n \pi]$, we obtain (18) with $\mu_{0}, \lambda_{1}$ given by (19) with $p=2$. Substituting (18) into (13) 
and integrating over $[0, \theta] \subset[0,2 n \pi]$, we obtain

$$
\begin{aligned}
r(\theta) & =r_{0}+\mu_{0}\left(t_{0}, \theta\right)+\mu_{1}\left(t_{0}, \theta\right) r_{0}^{-1}+O\left(r_{0}^{-2}\right) \\
t(\theta) & =t_{0}+\frac{\theta}{n}+\lambda_{1}\left(t_{0}, \theta\right) r_{0}^{-1}+\lambda_{2}\left(t_{0}, \theta\right) r_{0}^{-2}+O\left(r_{0}^{-3}\right) \\
r^{-1}(\theta) & =r_{0}^{-1}-\mu_{0}\left(t_{0}, \theta\right) r_{0}^{-2}+\left(\mu_{0}^{2}\left(t_{0}, \theta\right)-\mu_{1}\left(t_{0}, \theta\right)\right) r_{0}^{-3}+O\left(r_{0}^{-4}\right),
\end{aligned}
$$

where

$$
\begin{aligned}
\mu_{1}(t, \theta)= & \frac{1}{n} \int_{0}^{\theta} S^{\prime}\left(\frac{\tau}{n}\right) h^{\prime}\left(t+\frac{\tau}{n}\right) \lambda_{1}(t, \tau) d \tau \\
& +\frac{1}{n} \int_{0}^{\theta} S\left(\frac{\tau}{n}\right) S^{\prime}\left(\frac{\tau}{n}\right) h^{2}\left(t+\frac{\tau}{n}\right) d \tau \\
= & \frac{1}{n^{2}} \int_{0}^{\theta} S^{\prime}\left(\frac{\tau}{n}\right) h^{\prime}\left(t+\frac{\tau}{n}\right) \int_{0}^{\tau} S(u / n) h(t+u / n) d u d \tau \\
& +\frac{1}{n} \int_{0}^{\theta} S\left(\frac{\tau}{n}\right) S^{\prime}\left(\frac{\tau}{n}\right) h^{2}\left(t+\frac{\tau}{n}\right) d \tau
\end{aligned}
$$

and

$$
\begin{aligned}
\lambda_{2}(t, \theta)= & -\frac{1}{n} \int_{0}^{\theta} S\left(\frac{\tau}{n}\right) h\left(t+\frac{\tau}{n}\right) \mu_{0}(t, \tau) d \tau+\frac{1}{n} \int_{0}^{\theta} S^{2}\left(\frac{\tau}{n}\right) h^{2}\left(t+\frac{\tau}{n}\right) d \tau \\
& +\frac{1}{n} \int_{0}^{\theta} S(\tau) h(t+\tau) \lambda_{1}(t, \tau) d \tau \\
= & \frac{1}{n^{2}} \int_{0}^{\theta} S h \int_{0}^{\tau} S h^{\prime} d u d \tau+\frac{1}{n^{2}} \int_{0}^{\theta} S h^{\prime} \int_{0}^{\tau} S h d u d \tau \\
= & \frac{1}{n^{2}} \int_{0}^{\theta} S\left(\frac{\tau}{n}\right) h\left(t+\frac{\tau}{n}\right) d \tau \int_{0}^{\theta} S\left(\frac{\tau}{n}\right) h^{\prime}\left(t+\frac{\tau}{n}\right) d \tau
\end{aligned}
$$

Substituting (21)-(23) into (13) again and integrating over [0, 2n $]$, we obtain

$$
\begin{aligned}
& r_{1}=r_{0}+\mu_{0}\left(t_{0}\right)+\mu_{1}\left(t_{0}\right) r_{0}^{-1}+O\left(r_{0}^{-2}\right) \\
& t_{1}=t_{0}+2 \pi+\lambda_{1}\left(t_{0}\right) r_{0}^{-1}+\lambda_{2}\left(t_{0}\right) r_{0}^{-2}+\lambda_{3}\left(t_{0}\right) r_{0}^{-3}+O\left(r_{0}^{-4}\right),
\end{aligned}
$$

where $\lambda_{k}(t)=\lambda_{k}(t, 2 n \pi), k=1,2, \mu_{i}(t)=\mu_{i}(t, 2 n \pi), i=0,1$, and

$$
\begin{aligned}
\lambda_{3}(t)= & \frac{1}{n} \int_{0}^{2 n \pi} S^{3} h^{3} d \theta-\frac{2}{n} \int_{0}^{2 n \pi} S^{2} h^{2} \mu_{0} d \theta+\frac{2}{n} \int_{0}^{2 n \pi} S^{2} h h^{\prime} \lambda_{1} d \theta \\
& +\frac{1}{n} \int_{0}^{2 n \pi} S h\left(\mu_{0}^{2}-\mu_{1}\right) d \theta-\frac{1}{n} \int_{0}^{2 n \pi} S h^{\prime} \lambda_{1} \mu_{0} d \theta \\
& +\frac{1}{n} \int_{0}^{2 n \pi} S h^{\prime \prime} \lambda_{1}^{2} d \theta+\frac{1}{n} \int_{0}^{2 n \pi} S h^{\prime} \lambda_{2} d \theta .
\end{aligned}
$$


Now, substituting the expressions of $\mu_{0}, \mu_{1}, \lambda_{1}$ and $\lambda_{2}$ into (24) and using $\lambda_{1}(t) \equiv 0$, we obtain from Lemma 2 that $\lambda_{2}(t) \equiv 0$ and $\mu_{0}(t)=-\lambda_{1}(t) \equiv 0$. After some elementary calculation, we obtain the expression of $\lambda_{3}(t)$ given in Theorem 2.

\section{Proof of the theorems}

Now, we are ready to prove the main results of this paper.

Proof of Theorem 1. Assume the conditions of Theorem 1 hold. If (I) is satisfied, then the Poincaré map $P:\left(t_{0}, r_{0}\right) \rightarrow\left(t_{1}, r_{1}\right)$ of the solutions of (13) has the following form:

$$
\begin{aligned}
& t_{1}=t_{0}+2 \pi_{p}+\lambda_{1}\left(t_{0}\right) r_{0}^{-1}+O\left(r_{0}^{-2}\right) \\
& r_{1}=r_{0}+\mu_{0}\left(t_{0}\right)+O\left(r_{0}^{-1}\right),
\end{aligned}
$$

with $\mu_{0}(t)=-(p-1) \lambda_{1}^{\prime}(t)$.

Now we introduce another action variable $u$ and a positive parameter $\varepsilon$ by $r=\frac{1}{u \varepsilon}$ with $u \in[1,2]$. Then $r \gg 1 \Leftrightarrow \varepsilon \ll 1$. Under this transformation, (25) is changed to the following form:

$$
\begin{aligned}
t_{1} & =t_{0}+2 \pi_{p}+\lambda_{1}\left(t_{0}\right) u_{0} \varepsilon+O\left(\varepsilon^{2}\right) \\
u_{1} & =u_{0}-\mu_{0}\left(t_{0}\right) u_{0}^{2} \varepsilon+O\left(\varepsilon^{2}\right) .
\end{aligned}
$$

Let $t_{1}=t_{0}+\varepsilon R\left(t_{0}, u_{0}, \varepsilon\right), u_{1}=u_{0}+\varepsilon W\left(t_{0}, u_{0}, \varepsilon\right)$, then $R(t, u, \varepsilon)=\lambda_{1}(t) u+$ $O(\varepsilon), W(t, u, \varepsilon)=-\mu_{0}(t) u_{0}^{2}+O(\varepsilon)$, and for $t \in\left[0,2 n \pi_{p}\right], u \in[1,2]$, we have

$$
|R(t, u, \varepsilon)|+\left|\frac{\partial R(t, u, \varepsilon)}{\partial t}\right|+\left|\frac{\partial R(t, u, \varepsilon)}{\partial u}\right| \leq C_{1}
$$

and

$$
|W(t, u, \varepsilon)|+\left|\frac{\partial W(t, u, \varepsilon)}{\partial t}\right|+\left|\frac{\partial W(t, u, \varepsilon)}{\partial u}\right| \leq C_{2}
$$

for some constants $C_{1}, C_{2}$. Moreover, if $\min _{t \in \mathbb{R}} \lambda_{1}(t)=d_{0}>0$, we have for $\varepsilon \ll 1, t \in \mathbb{R}, u \in[1,2]$,

$$
\frac{\partial R(t, u, \varepsilon)}{\partial u} \geq \frac{d_{0}}{2}>0
$$

and if $\max _{t \in S^{1}} \lambda_{1}(t)=-d_{1}<0$, we have

$$
\frac{\partial R(t, u, \varepsilon)}{\partial u} \leq-\frac{d_{1}}{2}<0 .
$$


In both cases, the Poincaré map of (25) is a monotone map. Going back to (13), we know that the Poincaré map $Q:\left(\theta_{0}, r_{0}\right) \rightarrow\left(\theta_{1}, r_{1}\right)$ is also monotone if $r_{0} \gg 1$. Using similar arguments as in [11, Section 4], we may construct a map $\bar{Q}$ which is a global monotone twist homeomorphism of the cylinder $S^{1} \times \mathbb{R}$ and coincides with $Q$ on $S^{1} \times\left[A_{0},+\infty\right)$ with a fixed constant $A_{0} \gg 1$, where $S^{1}=\mathbb{R} / 2 \pi_{p} \mathbb{Z}$. Therefore, the existence of Mather sets $M_{\omega}$ of $\bar{Q}$ is guaranteed by Aubry-Mather theory (see [11]). Moreover, for some small $\varepsilon_{0}>0$, such invariant sets with rotation $\omega \in\left(n, n+\varepsilon_{0}\right)$ lie in the domain $S^{1} \times\left[A_{0},+\infty\right)$. Hence they are just the Aubry-Mather sets of the Poincare map of $Q$. The above discussion shows the existence of Mather sets, this implies that (1) has a solution $\left(x_{\omega}(t), x_{\omega}^{\prime}(t)\right)$ of Mather type. Moreover, if $\omega=\frac{m}{k}$ is a rational, the solutions $\left(x_{\omega}\left(t+2 i \pi_{p}\right), x_{\omega}^{\prime}\left(t+2 i \pi_{p}\right)\right), 1 \leq i \leq k-1$, are mutually unlinked periodic solutions of period $2 k \pi_{p}$ and $\lim _{k \rightarrow+\infty} \min _{t \in \mathbb{R}}\left\|\left(x_{\omega}(t), x_{\omega}^{\prime}(t)\right)\right\|=+\infty$. If $\omega$ is irrational, the solution $\left(x_{\omega}(t), x_{\omega}^{\prime}(t)\right)$ is either a usual quasi-periodic solution or a generalized one.

In case (II), by Lemma 1, the Poincaré map of (13) has the form of (14), under the same transformation $r=\frac{1}{u \varepsilon}$, (14) is of the following form:

$$
\begin{aligned}
t_{1} & =t_{0}+2 \pi_{p}+\varepsilon R_{1}\left(t_{0}, u_{0}, \varepsilon\right) \\
u_{1} & =u_{0}+\varepsilon W_{1}\left(t_{0}, u_{0}, \varepsilon\right)
\end{aligned}
$$

where $R_{1}(t, u, \varepsilon)=\lambda_{1}(t) u+\lambda_{1+\sigma} u^{1+\sigma} \varepsilon^{\sigma}+O\left(\varepsilon^{1}\right)$, and $W_{1}(t, u, \varepsilon)=-\mu_{0}(t) u^{2}+O(\varepsilon)$. It is easy to see that $R_{1}$ and $W_{1}$ satisfy the similar inequalities as (26) and (27). Moreover, for $\lambda_{1}(t) \geq 0$ and $D_{0}<0$, we have for $\varepsilon \ll 1, t \in \mathbb{R}, u \in[1,2], \lambda_{1+\sigma}>0$ and

$$
\frac{\partial R_{1}(t, u, \varepsilon)}{\partial u}=\lambda_{1}(t)+(1+\sigma) \lambda_{1+\sigma} \varepsilon^{\sigma}+O\left(\varepsilon^{1}\right) \geq \lambda_{1}(t)+\frac{1}{2}(1+\sigma) \lambda_{1+\sigma} \varepsilon^{\sigma}>0 .
$$

Similarly, for $\lambda_{1}(t) \leq 0$ and $D_{0}>0$, we have $\lambda_{1+\sigma}<0$ and

$$
\frac{\partial R_{1}(t, u, \varepsilon)}{\partial u}=\lambda_{1}(t)+(1+\sigma) \lambda_{1+\sigma} \varepsilon^{\sigma}+O\left(\varepsilon^{1}\right) \leq \lambda_{1}(t)+\frac{1}{2}(1+\sigma) \lambda_{1+\sigma} \varepsilon^{\sigma}<0 .
$$

The rest proof is similar to that of case (I), so we omit it for simplicity.

Proof of Theorem 2 (a sketch). By Lemma 2 and Lemma 3, the Poincaré map of (13) has the form of (17) or the form of (20). Under the transformation $r=\frac{1}{u \varepsilon},(17)$ and (20) have the forms

$$
\begin{aligned}
& t_{1}=t_{0}+2 \pi_{p}+\lambda_{2}\left(t_{0}\right) u_{0}^{2} \varepsilon^{2}+O\left(\varepsilon^{3}\right) \\
& u_{1}=u_{0}-\mu_{1}\left(t_{0}\right) u_{0}^{3} \varepsilon^{2}+O\left(\varepsilon^{3}\right)
\end{aligned}
$$


and

$$
\begin{aligned}
& t_{1}=t_{0}+2 \pi+\lambda_{3}\left(t_{0}\right) u_{0}^{3} \varepsilon^{3}+O\left(\varepsilon^{4}\right) \\
& u_{1}=u_{0}-\mu_{1}\left(t_{0}\right) u_{0}^{3} \varepsilon^{3}+O\left(\varepsilon^{4}\right),
\end{aligned}
$$

respectively. Let

$$
\begin{array}{lll}
t_{1}=t_{0}+2 \pi_{p}+\varepsilon^{2} R_{2}(t, u, \varepsilon), & u_{1}=u_{0}+\varepsilon^{2} W_{2}(t, u, \varepsilon) & \text { for } p \neq 2 \\
t_{1}=t_{0}+2 \pi+\varepsilon^{3} R_{3}(t, u, \varepsilon), & u_{1}=u_{0}+\varepsilon^{3} W_{3}(t, u, \varepsilon) & \text { for } p=2,
\end{array}
$$

respectively, then it is not difficult to verify that for $0<\varepsilon \ll 1, \frac{\partial R_{k}(t, u, \varepsilon)}{\partial u} \neq 0$ if $\lambda_{k}(t) \neq 0, t \in \mathbb{R}$ for $k=2,3$. The rest proofs are similar to that of Theorem 1 , so we omit them for simplicity.

Example 1. Consider equation (1) with $\alpha=\beta=n=1, h(t) \equiv 1, g(x)=$ $\arctan x+|x|^{-\tau} \operatorname{sgn} x$, where $\tau \in(0,1)$. Then Theorem 1 implies that, for all $t \in \mathbb{R}, \lambda_{1}(t)=-\frac{2 \pi}{p} B\left(\frac{1}{p}, \frac{1}{q}\right)<0$. Now (I) of Theorem 1 implies that the conclusion of Theorem 1 holds.

Example 2. Consider the following equation

$$
(p-1)^{-1}\left(\phi_{p}\left(x^{\prime}\right)\right)^{\prime}+\phi_{p}(x)+|x|^{-\tau} \operatorname{sgn} x-2|x|^{-\tau} \operatorname{sgn} x=1,
$$

where $p>1, \tau \in(0,1)$. Then $\alpha=\beta=n=1, c^{+}=1, c^{-}=-2, h(t) \equiv 1$, and it is easy to see that $S(t)=\sin _{p} t, \lambda_{1}(t) \equiv 0$ and $D_{0}=c^{+}+c^{-}<0$. Now (II) of Theorem 1 implies that there exists $\varepsilon_{0}>0$ such that for any $\omega \in\left(n, n+\varepsilon_{0}\right)$, (28) has a solution $\left(x_{\omega}(t), x_{\omega}^{\prime}(t)\right)$ of Mather type with rotation number $\omega$.

Example 3. Consider a special case of (1):

$$
(p-1)^{-1}\left(\phi_{p}\left(x^{\prime}\right)\right)^{\prime}+\phi_{p}(x)=1 .
$$

In this example, $p \neq 2, \alpha=\beta=n=1, g(x) \equiv 0, h(t)=1$. Then it can be verified that $\lambda_{1}(t) \equiv 0, \lambda_{2}(t)=(2-p) \int_{0}^{2 \pi_{p}} \sin _{p}^{2} \theta d \theta \neq 0$. Now Theorem 2 implies that there exists $\varepsilon_{0}>0$ such that for any $\omega \in\left(n, n+\varepsilon_{0}\right)$, (29) has a solution $\left(x_{\omega}(t), x_{\omega}^{\prime}(t)\right)$ of Mather type with rotation number $\omega$.

Example 4. Consider the following linear equation

$$
x^{\prime \prime}+\alpha x^{+}-\beta x^{-}=h(t),
$$

where $\alpha \neq \beta$ satisfying (2) with $p=2, n=1$, and $h$ is piecewise continuous and $2 \pi$-periodic such that $h(t)=1, t \in\left[0, \frac{\pi}{\sqrt{\alpha}}\right] ; h(t)=\frac{\beta}{\alpha}, t \in\left(\frac{\pi}{\sqrt{\alpha}}, 2 \pi\right]$. Then it follows from Theorem 2 that $\lambda_{1}(t)=\lambda_{2}(t) \equiv 0$ and $\lambda_{3}(t) \equiv \lambda_{3}(0)=-\frac{2}{3 \alpha^{3}}(\alpha-\beta)$ $\neq 0$. Hence Theorem 2 implies that the conclusion of Theorem 2 holds.

Remark 1. Let $p=2$, Theorem 1 reduces to [3, Theorem 1], moreover, our assumption $D_{0} \neq 0$ is weaker than the assumption $c^{ \pm} \neq 0$ and $c^{+} c^{-}>0$. In case $g(x) \equiv 0$ and $\lambda_{1}(t) \equiv 0$, the result of [3] cannot be applied to equation (30), but Theorem 2 gives partial results. Therefore, our results are natural generalization and refinements of the result of [3]. 


\section{References}

[1] Alonso, J. M. and Ortega, R., Unbounded solutions of semilinear equations at resonance. Nonlinearity 9 (1996), 1099 - 1111.

[2] Alonso, J. M. and Ortega, R., Roots of unity and unbounded motions of an asymmetric oscillator. J. Diff. Equations 143 (1998), 201 - 220.

[3] Capietto, A. and Liu, B., Quasi-periodic solutions of a forced asymmetric oscillator at resonance. Nonlinear Anal. 56 (2004), 105 - 117.

[4] Fabry, C. and Fonda, A., Nonlinear resonance in asymmetric oscillators. J. Diff. Equations 147 (1998), 58 - 78.

[5] Fabry, C. and Mawhin, J., Oscillations of a forced asymmetric oscillator at resonance. Nonlinearity 13 (2000), 493 - 505.

[6] Fonda, A., Positively homogeneous hamiltonian systems in the plane. J. Diff. Equations 200 (2004), 162 - 184.

[7] Liu, B., Boundedness in asymmetric oscillations. J. Math. Anal. Appl. 231 (1999), $355-373$.

[8] Liu, B. and You, J., Quasiperiodic solutions of Duffing's equations. Nonlinear Anal. 33 (1998), 645 - 655.

[9] Ortega, R., Boundedness in a piece-wise linear oscillator and a variant of the small twist theorem. Proc. London Math. Soc. 79 (1999), $381-413$.

[10] Pino, M. A., Drabek, P. and Manasevich, R., The Fredholm alternative at the first eigenvalue for the one dimensional p-Laplacian. J. Diff. Equations 151 (1999), $355-373$.

[11] Pei, M., Aubry-Mather sets for finite-twist maps of a cylinder and semilinear Duffing equations. J. Diff. Equations 113 (1994), 106 - 127.

[12] Yang, X., Boundedness in nonlinear asymmetric oscillations. J. Diff. Equations 183 (2002), $108-131$.

[13] Yang, X., Boundedness of solutions of a class of nonlinear systems. Math. Proc. Cambridge Phil. Soc. 136 (2004), 185 - 193.

[14] Yang, X., Boundedness in nonlinear oscillations. Math. Nachr. 128 (2004), $102-113$.

[15] Yuan, X., Lagrange stability for asymmetric Duffing equations. Nonlinear Anal. 43 (2001), $137-151$.

Received December 28, 2004; revised September 7, 2005 